\title{
Real-time estimation method of roll angle based on least squares recursion
}

\author{
Chen Jingsheng ${ }^{1, *}, \mathrm{Li}$ Chuanjun ${ }^{1}$, and $H u$ Peisen $^{1}$ \\ ${ }^{1}$ School of Aerospace Engineering, Beijing Institute of Technology, Beijing, China
}

\begin{abstract}
The rotary missile stands a high overload during the launch and has to be powered up after launch, so it is necessary to achieve inflight alignment under high dynamic conditions. As a key technology of inflight alignment, the measurement method of roll angle has attracted more and more attention from researchers. The rotational speed of the rotary missile is very high, and most MIMUs cannot directly measure the roll angle. To solve this problem, this paper proposes a roll angle estimation method based on least squares method, analyzes its principle and derives the calculation procedure. Then on this basis, the roll angle estimation method based on least squares recursion is studied. The principle and calculation procedure of this method are deduced in detail. At last, the simulation experiment on MATLAB is carried out. The results show that this method is simple in calculation, high in accuracy and good in real-time performance, and has great application value.
\end{abstract}

\section{Introduction}

With the advancement of science and technology, modern warfare demands increasingly higher on the accuracy of weapon systems. For the guided weapons that use Strapdown Inertial Navigation (SINS) and Satellite Navigation (GNSS) integrated navigation, improving the initial alignment accuracy is an effective means to improve the system's strike accuracy. The GNSS/SINS integrated navigation system usually has to withstand high overload and high speed during the launching. At this time, both SINS and GNSS cannot work normally. Therefore, the SINS has to achieve inflight alignment under the stable or low-speed rotation conditions after the missile enters the controlled section[1].

Because of the high overload, SINS cannot be powered up at the moment the rotary missile is fired. During the launch, the missile is rolling in the cannon. The state and the roll angle of the missile is unknown after the launch, which makes the difficulty to the initial alignment of the SINS. According to the ballistic data of the short time after the missile exits the cannon, the position, velocity, yaw angle and pitch angle of the SINS can be initially set before the launch with the previously known launch conditions[2][3]. However, the roll angle cannot be directly measured, so the estimation of the initial roll angle has been widely concerned.

In order to solve this problem, this paper proposes a roll angle estimation method based on least squares method[4], using gyro data and GNSS data to estimate the roll angle. And on this basis, a real-time roll angle estimation method based on least squares recursion is proposed. This paper analyzes the principles and derive the calculation procedure. At last, a simulation experiment is carried out to verify the performance of the algorithm. The results of the experiment show that this method has high accuracy of roll angle and good performance in high rotation environment.

\section{Roll Angle Estimation Based On Least Squares Method}

This paper mainly studies on roll angle estimation method for SINS/GNSS integrated navigation system, and the functional composition of the system is shown as Fig.1.

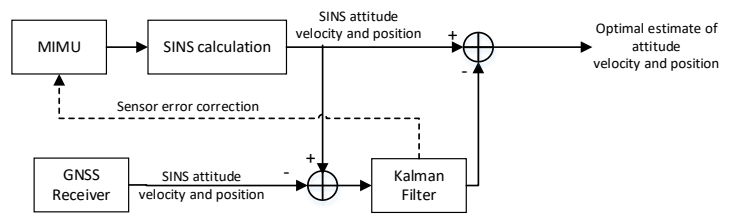

Fig. 1. Functional composition of the system

As we can see from the Fig.1, the SINS and GNSS calculate the attitude, velocity and position independently of each other, and then the results are integrated by a feedforward complementary Kalman filter to output the optimal estimate of the attitude, velocity and position. The results can help MIMU correct its own error and improve the accuracy. The placement of the sensors in MIMU is generally shown in Fig.2.

*Corresponding author: 1454341945@qq.com 


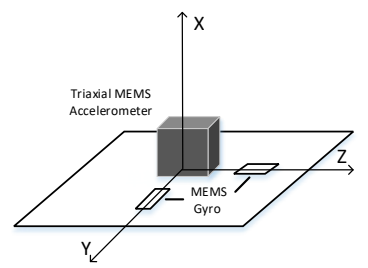

Fig. 2. The placement of the sensors in MIMU

During the flight of the missile, the $0 x_{1}$ axis of the missile body coordinate system and the $0 x_{4}$ axis of the missile body axis coordinate system keep coincident. The $0 y_{1}$ axis and the $0 z_{1}$ axis rotate with the missile body, and the $0 y_{4}$ axis keeps in the vertical plane. $0 z_{4}$ axis can be obtained from $0 x_{4}$ axis and $0 y_{4}$ axis by the right-hand system rule. The diagram of missile body coordinate system and missile body axis coordinate system is shown as Fig.3.

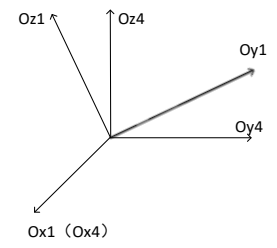

Fig. 3. The diagram of these two coordinate system

Therefore, we can get the conversion equation between the body axis coordinate system and the body coordinate system[5]:

$$
\left\{\begin{array}{c}
\omega_{x 1}=\omega_{x 4} \\
\omega_{y 1}=\omega_{y 4} \cos \gamma+\omega_{z 4} \sin \gamma \\
\omega_{z 1}=-\omega_{y 4} \sin \gamma+\omega_{z 4} \cos \gamma
\end{array}\right.
$$

In the formula, $\omega_{x 1} 、 \omega_{y 1} 、 \omega_{z 1}$ are the three-axis components of the body rotational angular velocity in the body coordinate system. $\omega_{x 4} 、 \omega_{y 4} 、 \omega_{z 4}$ are the three-axis components of the body rotational angular velocity in body axis coordinate system.

In the body axis coordinate system, the kinematics equation of the missile around the center of mass can be expressed as:

$$
\left\{\begin{array}{c}
\dot{\gamma}=\omega_{x 4}-\omega_{y 4} \tan \vartheta \\
\psi=\frac{1}{\cos \vartheta} \omega_{y 4} \\
\dot{\vartheta}=\omega_{z 4}
\end{array}\right.
$$

In the formula, $\gamma$ is the roll angle, $\psi$ is the yaw angle, and $\vartheta$ is the pitch angle. Substituting Equation (2) into Equation (1), the last two formulas of Equation (1) can be written as:

$$
\left\{\begin{array}{c}
\omega_{y 1}=(\psi \cos \vartheta) \cos \gamma+\dot{\vartheta} \sin \gamma \\
\omega_{z 1}=(-\psi \cos \vartheta) \sin \gamma+\dot{\vartheta} \cos \gamma
\end{array}\right.
$$

Organize it into a matrix, and get:

$$
\left[\begin{array}{c}
\omega_{y 1} \\
\omega_{z 1}
\end{array}\right]=\left[\begin{array}{cc}
\dot{\vartheta} & \psi \cos \vartheta \\
-\psi \cos \vartheta & \dot{\vartheta}
\end{array}\right] \cdot\left[\begin{array}{c}
\sin \gamma \\
\cos \gamma
\end{array}\right]
$$

In the formula, the values of $\vartheta 、 \dot{\vartheta}$ and $\psi$ can be approximately estimated by the GNSS velocity measurement data[6]. Substituting the corresponding values at each moment into Equation (4), a matrix equation of the Equation (5) is obtained.

$$
\boldsymbol{Z}_{2 n \times 1}=\boldsymbol{H}_{2 n \times 2} \cdot \boldsymbol{X}_{2 \times 1}
$$

Then the least squares estimation of $\boldsymbol{X}$ can be expressed as:

$$
\hat{\boldsymbol{X}}=\left(\boldsymbol{H}^{T} \boldsymbol{H}\right)^{-1} \boldsymbol{H}^{T} \boldsymbol{Z}
$$

And $\boldsymbol{X}$ can meet the requirement to make:

$$
J(\hat{\boldsymbol{X}})=(\boldsymbol{Z}-\boldsymbol{H} \hat{\boldsymbol{X}})^{T}(\boldsymbol{Z}-\boldsymbol{H} \hat{\boldsymbol{X}})=\min
$$

Calculate the estimated value of $\sin \gamma$ and $\cos \gamma$ from Equation (6), then:

$$
\gamma=\arctan \left(\frac{\sin \gamma}{\cos \gamma}\right)
$$

The range of values is determined by Equation (9).

$$
\left\{\begin{array}{cc}
\text { when } \sin \gamma \leq 0 \text { and } \cos \gamma<0, & 180^{\circ} \leq \gamma<270^{\circ} \\
\text { when } \sin \gamma \leq 0 \text { and } \cos \gamma>0, & 270^{\circ} \leq \gamma<360^{\circ} \\
\text { when } \sin \gamma \geq 0 \text { and } \cos \gamma<0, & 90^{\circ} \leq \gamma<180^{\circ} \\
\text { when } \sin \gamma \geq 0 \text { and } \cos \gamma>0, & 0^{\circ} \leq \gamma<90^{\circ} \\
\text { when } \sin \gamma>0 \text { and } \cos \gamma=0, & \gamma=90^{\circ} \\
\text { when } \sin \gamma<0 \text { and } \cos \gamma=0, & \gamma=270^{\circ}
\end{array}\right.
$$

Therefore, according to this method, based on the collected GNSS data, the corresponding pitch angle and yaw angle and their rate of change are estimated, and combined with the measurement data of the MIMU, the roll angle of the rotary missile at each moment can be estimated.

\section{Real-Time Estimation Of Roll Angle Based On Least Squares Recursion}

In the previous chapter, the roll angle estimation method based on least squares method was studied, but there exist some defects of this method. The least squares method has to be processed after all the data has been collected. When estimating the roll angle using the least squares method, it is necessary to let the missile fly for a period of time, and collect the MIMU and GNSS data during this time, and then use the least squares method to do the estimation, so that the measurement data cannot be processed in time. And each time using the least squares estimation, all the previously processed data need to be recalculated.

In order to solve this problem, this paper proposes a real-time estimation method of roll angle based on least squares recursion. This method can process the collected data in time after obtaining the gyro and GNSS data, without waiting for all the measurement data to be obtained after measurement. And when estimating the roll angle of the current time, the previous result can be utilized without calculating previously processed data again, and it saves the computation time very much.

It is assumed that the $k^{-1}$ th time estimation result has been obtained when the least squares estimation is performed at the $\mathrm{k}$ th time.

$$
\hat{\boldsymbol{X}}_{k-1}=\left(\boldsymbol{H}_{k-1}^{T} \boldsymbol{H}_{k-1}\right)^{-1} \boldsymbol{H}_{k-1}^{T} \boldsymbol{Z}_{k-1}
$$


In the formula, $\boldsymbol{Z}_{k-1} 、 \boldsymbol{H}_{k-1}$ can be expressed as:

$$
\boldsymbol{Z}_{k-1}=\left(\begin{array}{l}
\boldsymbol{Z}(1) \\
\boldsymbol{Z}(2) \\
\vdots \\
\boldsymbol{Z}(k-1)
\end{array}\right), \boldsymbol{H}_{k-1}=\left(\begin{array}{l}
\boldsymbol{H}(1) \\
\boldsymbol{H}(2) \\
\vdots \\
\boldsymbol{H}(k-1)
\end{array}\right)
$$

Assume $\boldsymbol{M}_{k-1}=\left(\boldsymbol{H}_{k-1}^{T} \boldsymbol{H}_{k-1}\right)^{-1}$, then

$$
\hat{\boldsymbol{X}}_{k-1}=\boldsymbol{M}_{k-1} \boldsymbol{H}_{k-1}^{T} \boldsymbol{Z}_{k-1}
$$

When performing the least squares estimation at the $\mathrm{k}$ th time, $\boldsymbol{H}(k)$ can be the estimated by GNSS velocity measurement data, and $\boldsymbol{Z}(k)$ can be obtained from the gyro data. According to the least squares method, get Equation (13).

$$
\hat{\boldsymbol{X}}_{k}=\left(\boldsymbol{H}_{k}^{T} \boldsymbol{H}_{k}\right)^{-1} \boldsymbol{H}_{k}^{T} \boldsymbol{Z}_{k}
$$

According to Equation (11), $\boldsymbol{Z}_{k}$ and $\boldsymbol{H}_{k}$ can be expressed as:

$$
\boldsymbol{Z}_{k}=\left(\begin{array}{l}
\boldsymbol{Z}(1) \\
\boldsymbol{Z}(2) \\
\vdots \\
\boldsymbol{Z}(k)
\end{array}\right)=\left(\begin{array}{l}
\boldsymbol{Z}_{k-1} \\
\boldsymbol{Z}(k)
\end{array}\right), \boldsymbol{H}_{k}=\left(\begin{array}{l}
\boldsymbol{H}(1) \\
\boldsymbol{H}(2) \\
\vdots \\
\boldsymbol{H}(k)
\end{array}\right)=\left(\begin{array}{l}
\boldsymbol{H}_{k-1} \\
\boldsymbol{H}(k)
\end{array}\right)
$$

Assume $\boldsymbol{M}_{k}=\left(\boldsymbol{H}_{k}^{T} \boldsymbol{H}_{k}\right)^{-1}$, then

$$
\begin{aligned}
& \boldsymbol{M}_{k}=\left[\left(\boldsymbol{H}_{k-1}^{T} \boldsymbol{H}^{T}(k)\right)\left(\begin{array}{l}
\boldsymbol{H}_{k-1} \\
\boldsymbol{H}(k)
\end{array}\right)\right]^{-1} \\
& =\left[\boldsymbol{H}_{k-1}^{T} \boldsymbol{H}_{k-1}+\boldsymbol{H}^{T}(k) \boldsymbol{H}(k)\right]^{-1}
\end{aligned}
$$

Substituting $\boldsymbol{M}_{k-1}=\left(\boldsymbol{H}_{k-1}^{T} \boldsymbol{H}_{k-1}\right)^{-1}$ into Equation (15), get Equation (16).

$$
\boldsymbol{M}_{k}=\left[\boldsymbol{M}_{k-1}^{-1}+\boldsymbol{H}^{T}(k) \boldsymbol{H}(k)\right]^{-1}
$$

After matrix inversion, get Equation (17):

$$
\boldsymbol{M}_{k}^{-1}=\boldsymbol{M}_{k-1}^{-1}+\boldsymbol{H}^{T}(k) \boldsymbol{H}(k)
$$

It can be seen from the Equation (17) that when $\boldsymbol{M}_{k-1}$ is definite, $\boldsymbol{M}_{k}$ can be obtained based on $\boldsymbol{H}(k)$.

According to Equation (13) and Equation (14):

$$
\begin{aligned}
& \hat{\boldsymbol{X}}_{k}=\boldsymbol{M}_{k} \boldsymbol{H}_{k}^{T} \boldsymbol{Z}_{k}=\boldsymbol{M}_{k}\left(\boldsymbol{H}_{k-1}^{T} \boldsymbol{H}^{T}(k)\right)\left(\begin{array}{c}
\boldsymbol{Z}_{k-1} \\
\boldsymbol{Z}(k)
\end{array}\right) \\
& =\boldsymbol{M}_{k}\left[\boldsymbol{H}_{k-1}^{T} \boldsymbol{Z}_{k-1}+\boldsymbol{H}^{T}(k) \boldsymbol{Z}(k)\right]
\end{aligned}
$$

Pre-multiply the both sides of Equation (12) by $\boldsymbol{M}_{k-1}^{-1}$, and get Equation (19) .

$$
\boldsymbol{M}_{k-1}^{-1} \hat{\boldsymbol{X}}_{k-1}=\boldsymbol{H}_{k-1}^{T} \boldsymbol{Z}_{k-1}
$$

Substituting Equation (19) into Equation (18),

$\hat{\boldsymbol{X}}_{k}=\boldsymbol{M}_{k}\left[\boldsymbol{M}_{k-1}^{-1} \hat{\boldsymbol{X}}_{k-1}+\boldsymbol{H}^{T}(k) \boldsymbol{Z}(k)\right]$

Substituting Equation (17) into Equation (20),

$$
\begin{aligned}
& \hat{\boldsymbol{X}}_{k}=\boldsymbol{M}_{k}\left[\left(\boldsymbol{M}_{k}^{-1}-\boldsymbol{H}^{T}(k) \boldsymbol{H}(k)\right) \hat{\boldsymbol{X}}_{k-1}+\boldsymbol{H}^{T}(k) \boldsymbol{Z}(k)\right] \\
& =\hat{\boldsymbol{X}}_{k-1}-\boldsymbol{M}_{k} \boldsymbol{H}^{T}(k) \boldsymbol{H}(k) \hat{\boldsymbol{X}}_{k-1}+\boldsymbol{M}_{k} \boldsymbol{H}^{T}(k) \boldsymbol{Z}(k) \\
& =\hat{\boldsymbol{X}}_{k-1}+\boldsymbol{M}_{k} \boldsymbol{H}^{T}(k)\left[\boldsymbol{Z}(k)-\boldsymbol{H}(k) \hat{\boldsymbol{X}}_{k-1}\right]
\end{aligned}
$$

Therefore, Equation (17) and Equation (21) constitute the least squares recursion formula. In this formula, $\hat{\boldsymbol{X}}_{k-1} 、 \boldsymbol{M}_{k-1}^{-1}$ are the results of the previous least squares estimation, $\boldsymbol{H}(k)$ and $\boldsymbol{Z}(k)$ can be obtained from the current measurement data. According to this method, measurement data can be processed in time, and the least squares estimation can be performed in real time without waiting for all the data to be obtained after measurement.

\section{Simulation Experiment Of Roll Angle Estimation}

Based on the last two chapters, Using MATLAB to simulate the roll angle estimation method based on least squares and least squares recursion[7]. The procedures of simulation are as follows:

(1) $5 \mathrm{~s}$ after the missile was launched, its flight state is stable and the angle of attack and angle of side slip are small. At this time, the gyro data of the MIMU and the GNSS receiver velocity data are collected. The current time data of the GNSS is used to calculate the pitch angle and the yaw angle of the missile and their rate of change. Then roll angle estimation is started.

(2) Combined with the results of the previous roll angle estimation, using the collected gyro data of the MIMU and the GNSS data to perform the least squares recursion estimation.

Through simulation experiments, the real-time estimation values of $\sin \gamma$ and $\cos \gamma$ are obtained as shown in the figure below, and the obvious phase difference can be seen from the figures.

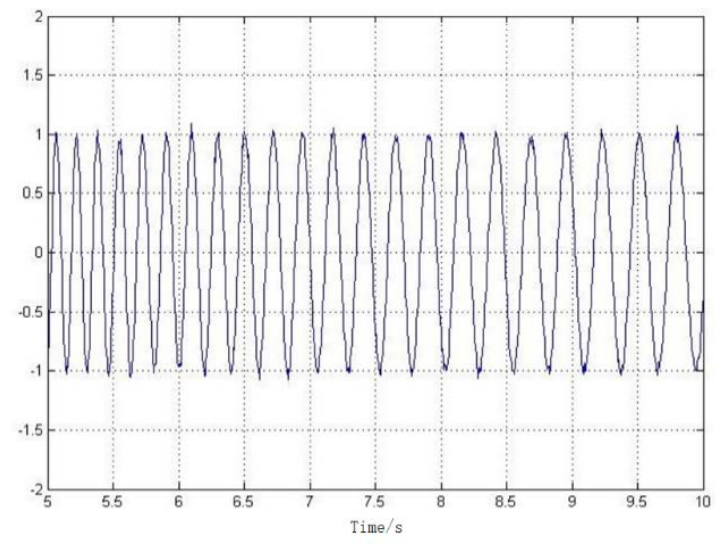

Fig. 4. The estimation values of $\sin \gamma$ 


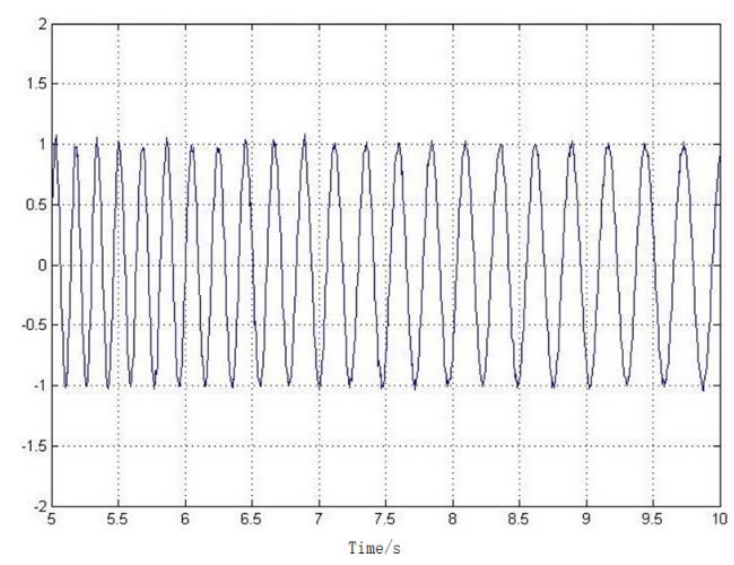

Fig. 5. The estimation values of $\cos \gamma$

According to Equation (9), the roll angle and its error of least squares recursion estimation can be obtained and shown as follow figures.

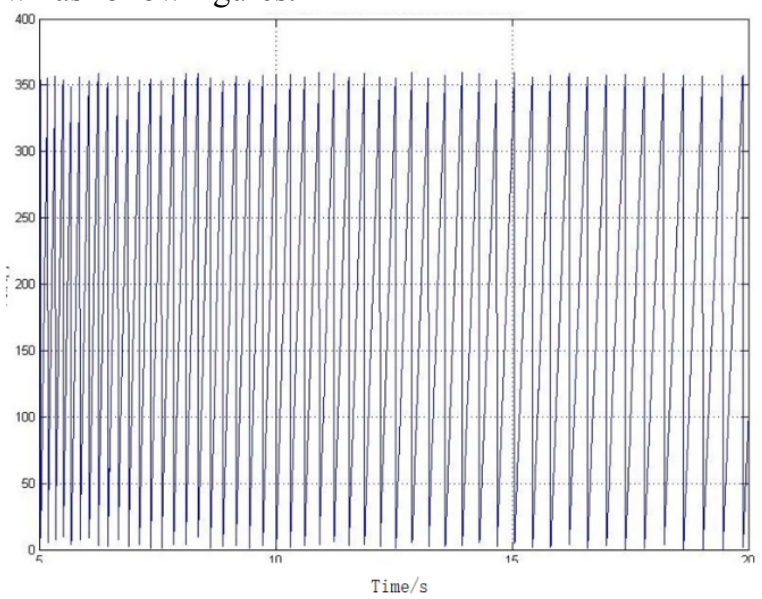

Fig. 6. Values of the roll angle real-time estimation

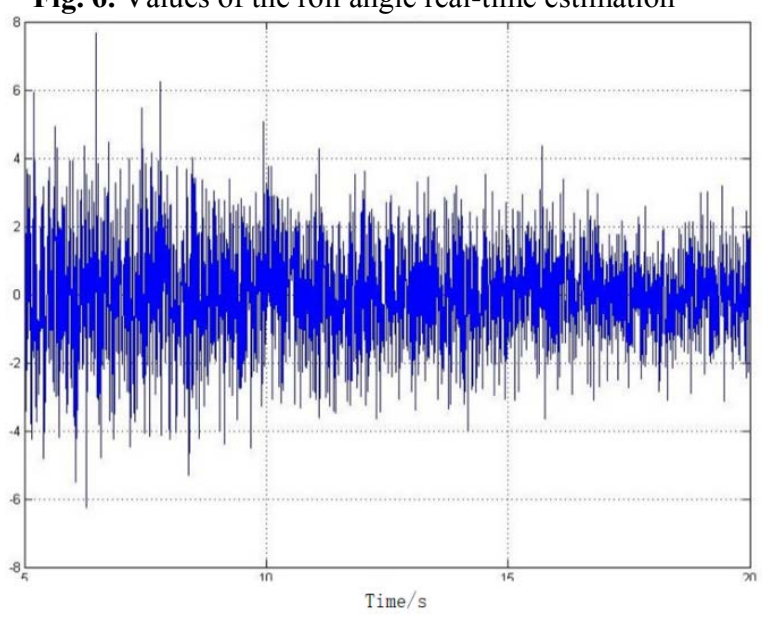

Fig. 7. Error of the roll angle real-time estimation

As can be seen from the Fig.6 and Fig.7, the roll angle error based on the least squares recursion estimation is stable throughout the flight time of the missile, and it fluctuates roughly within the interval of $\pm 4^{\circ}$ and the fluctuation amplitude becomes smaller and smaller, and finally remains at the $\pm 2^{\circ}$ interval. The result shows that the estimation accuracy of the roll angle meets the requirements of the coarse alignment of inertial navigation.

Using the gyro data of MIMU and GNSS velocity data collected in the simulation, the estimation based on least squares method is performed. Roll angle and its error are shown in the figures below.

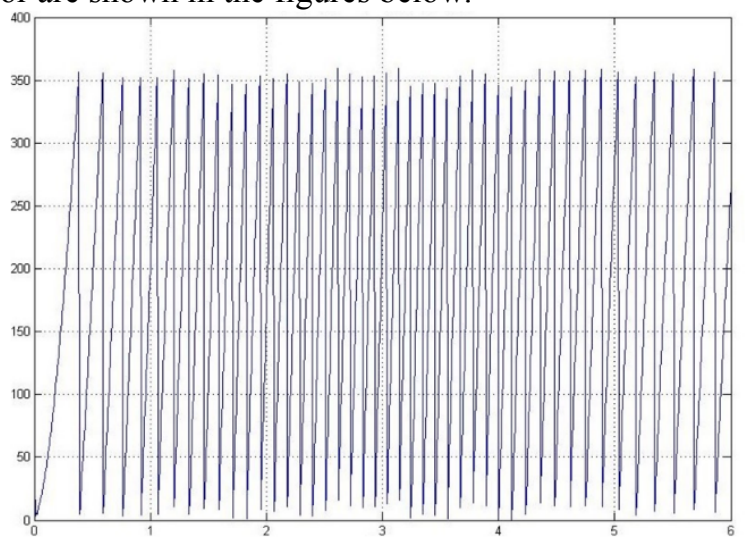

Fig. 8. Values of roll angle estimation

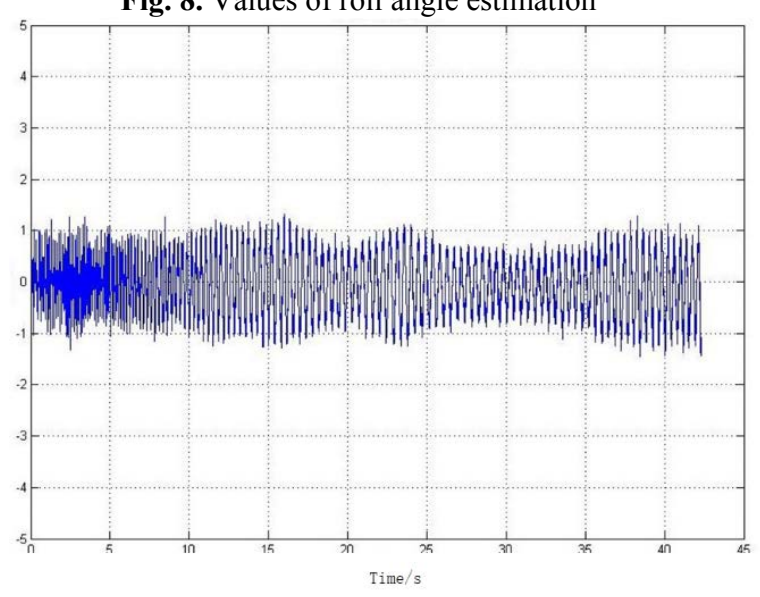

Fig. 9. Error of the roll angle estimation

It can be seen from the Fig. 8 and Fig.9 that the estimated roll angle error based on the least squares method is relatively stable throughout the flight time of the missile, and is generally in the interval of $\pm 1^{\circ}$. The result shows that this method is of high precision and worth to be studied.

Through the simulation experiment, we can see that the roll angle error estimated by the least squares recursion is larger than the roll angle error estimated by the least squares method after the measurement. However, the error keeps fluctuating within the range of $\pm 4^{\circ}$ and the fluctuation amplitude is getting smaller and smaller. The estimation method based on least squares recursion has better real-time performance, and the data can be processed online for estimation. This is a great advantage of this method.

\section{Conclusion}

Based on the least squares method, this paper proposes a roll angle estimation method based on least squares recursion, and do the simulation experiment for this method. The simulation results show that this method is 
simple in calculation, good in real-time preforms and high in accuracy. It meets the precision requirements of the strap-down inertial navigation system for coarse alignment under the high speed conditions and has great application value.

This paper is supported by National Natural Science Foundation of China (61473039) and the key laboratory of dynamics and control of flight vehicle, Ministry of education, school of aerospace engineering, Beijing Institute of Technology.

\section{References}

1. Yang Denghong. Research on initial alignment method of GNSS/MIMU integrated navigation for rotating stable missile [D]. Beijing: Beijing Institute of Technology, 2015.

2. She Haoping, Yang Shuxing, Ni Hui. A New Algorithm for Estimating the Initial Rolling Angle of GPS/INS Combined Missiles in Air Alignment[J]. , 2009, 32(10): 1265-1270.

3. Ma Chunyan, Liu Li, Du Xiaojing, et al. Determination of Initial Rolling Angle of Terminal Guided Projectile[J]. Journal of Projectiles, Missiles and Guidance, 2005, 25(s4): 356-358.

4. Liu Yukuan, Li Chuanjun, Chen Jingsheng. Rolling Angle Estimation Method for GNSS/SINS Integrated Navigation System Based on Least Square Method. ICCSSE Proceedings Volumes, 2018, pp.330-333.

5. Qin Yongyuan. Inertial Navigation [M]. Beijing: Science Press, 2014.

6. An Liangliang, Wang Liangming. GPS/SINS combined attitude measurement method for high-rotation rockets[J]. Journal of Ballistics, 2016, 28(1): 39-44.

7. Jin Xuebo. Theory and Application of Kalman Filter-Based on MATLAB[M]. Beijing: Science Press, 2016. 